\title{
The quantum gravitational black hole is neither black nor white
}

\author{
T. P. Singh ${ }^{a 1}$ and Cenalo Vaz ${ }^{b 2}$ \\ ${ }^{a}$ Tata Institute of Fundamental Research, \\ Homi Bhabha Road, Mumbai 400 005, India. \\ ${ }^{b}$ Department of Physics, University of Cincinnati \\ Cincinnati, Ohio, USA
}

\begin{abstract}
Understanding the end state of black hole evaporation, the microscopic origin of black hole entropy, the information loss paradox, and the nature of the singularity arising in gravitational collapse - these are outstanding challenges for any candidate quantum theory of gravity. Recently, a midisuperspace model of quantum gravitational collapse has been solved using a lattice regularization scheme. It is shown that the mass of an eternal black hole follows the Bekenstein spectrum, and a related argument provides a fairly accurate estimate of the entropy. The solution also describes a quantized massenergy distribution around a central black hole, which in the WKB approximation, is precisely Hawking radiation. The leading quantum gravitational correction makes the spectrum non-thermal, thus providing a plausible resolution of the information loss problem.
\end{abstract}

\footnotetext{
${ }^{1}$ e-mail address: tpsingh@tifr.res.in

${ }^{2}$ e-mail address: vaz@physics.uc.edu
} 
Recent developments in quantum general relativity have provided important insights into long-standing questions relating to the end-state of black hole evaporation, the origin of black-hole entropy, the information loss paradox and the fate of the singularities arising in classical gravitational collapse. These advances in our understanding of collapse have become possible because of a successful canonical quantization and lattice regularization of a midisuperspace model of quantum gravitational collapse [1. They are in keeping with the new understanding that has recently been achieved with regard to the cosmological singularity in loop quantum cosmology [2].

Following earlier pioneering work by Kuchař [3] and by Kastrup and Thiemann [4, we developed a canonical description of spherical dust collapse as described by the LemaitreTolman-Bondi models. For the marginally bound models, the canonical variables are the area radius $R$, the dust proper time $\tau$, the mass function, $F=2 M$, of the collapsing dust cloud and their conjugate momenta. The momentum conjugate to $F$ can be eliminated using the momentum-constraint, to obtain the following simple form for the Hamiltonian constraint [5]

$$
\left(P_{\tau}+F^{\prime} / 2\right)^{2}+\mathcal{F} P_{R}^{2}-\frac{F^{\prime 2}}{4 \mathcal{F}}=0,
$$

where $F^{\prime}$ is the derivative of $F(r)$ with respect to the comoving coordinate $r$ (it represents the shell mass density), and $\mathcal{F}=1-F / R$.

One can introduce a flat DeWitt metric on the configuration space by defining $R_{*}=$ $\int d R / \sqrt{|\mathcal{F}|}$. Then the quantum gravitational collapse corresponding to (11) is described by the Wheeler-DeWitt equation

$$
\left[\frac{1}{c^{2}} \frac{\delta^{2}}{\delta \tau^{2}(x)} \pm \frac{\delta^{2}}{\delta R_{*}^{2}(x)} \pm \frac{f^{\prime 2}}{4 l_{p}^{2}|\mathcal{F}|}\right] \Psi[\tau, R]=0,
$$

where we have used the dimensionless variables $x=r / l_{p}$, and $f(x)=F / l_{p}$. The upper sign refers to the region $R>F$, and the lower sign to $R<F$. The apparent horizon is the curve $R=F$. The momentum constraint is satisfied if the wave functional is a spatial scalar, which we assume to be of the "stationary state" form

$$
\Psi[\tau, R]=\exp \left[-\frac{i c}{2 l_{p}} \int_{0}^{\infty} d x f^{\prime}(x)(\tau+\mathcal{U}(R))\right] .
$$

To regularize the functional derivatives, we choose a lattice by dividing space into cells, the size of the $j^{\text {th }}$ cell being $\sigma_{j}$, and we finally take the limit $\sigma_{j} \rightarrow 0, \forall j$. Remarkably enough, the lattice size drops out of the Wheeler-DeWitt equation, which factors into an infinite set of ordinary differential equations for the time independent wave functions, one for each cell 
9]:

$$
z(z-1)^{2} \frac{d^{2} y}{d z^{2}}+\frac{z-1}{2} \frac{d y}{d z}+\gamma^{2} z^{2} y=0 .
$$

The quantities $y, z$ and $\gamma$ are defined independently for each cell, and for the $j^{\text {th }}$ cell are given by $z_{j}=R_{j} / F_{j}, \gamma_{j}=F_{j} \omega_{j} / c$ and $y_{j} \equiv \Psi_{j}(z)$, which is the time-independent wave function for the $j^{t h}$ cell. The frequency, $\omega_{j}$, defined by the relation

$$
f_{j+1}-f_{j} \equiv \frac{2 l_{p}}{c} \omega_{j}
$$

is related to the energy density, $\varepsilon_{j}$, of the $j^{\text {th }}$ shell by $\varepsilon_{j}=\hbar \omega_{j}$.

The simple form to which the canonical quantum dynamics of the collapsing cloud has been reduced pays off rich dividends. One is able to draw significant conclusions about the Bekenstein mass spectrum, black hole entropy, and quantum gravitational corrections to Hawking radiation.

An eternal black hole is described by a mass function $F(r)$ which is non-vanishing only at the origin (we recall that the Schwarzschild black hole is a special case of the Tolman-Bondi solution). In this case, the above midisuperspace problem reduces to quantum mechanics. The stationary states of the black hole are a superposition of ingoing and outgoing waves in the interior and in the exterior they are exponentially decaying because the Wheeler-Dewitt equation is elliptic when $R>F$. Matching the wave function and its derivative at the horizon directly yields the Bekenstein mass spectrum [6]

$$
M^{2}=\left(n+\frac{1}{2}\right) M_{p}^{2} .
$$

Insight into the origin of black hole entropy can be obtained by taking a mass function $F=2 M$ that describes a series of successive collapsing shells, each of which obeys a mass quantization condition analogous to (6),

$$
\mu_{j} M_{j}=\left(n_{j}+\frac{1}{2}\right) M_{p}^{2}
$$

where $\mu_{j}$ is the mass of the $j^{\text {th }}$ shell and $M_{j}$ is the mass contained within it. These conditions, when applied recursively, show that the mass of the $j^{\text {th }}$ shell is determined by $j$ quantum numbers. Thus the total mass of a quantum black hole formed by $N$ collapsed shells will depend on $N$ quantum numbers and a quantum black hole cannot be described simply by its total mass because such a description would ignore the manner in which the mass was distributed among the shells. The entropy counts the number of distributions for a given total mass. For an eternal black hole, $M_{k}$ in (7) should be replaced by $M$, the mass of the 
hole. The total mass (squared) of the hole continues quantized as before and the problem of counting the number of distributions is precisely the problem of asking for the number of ways in which $N$ integers may be added to give another integer. This result depends on the number of shells that have collapsed to form the black hole, which we do not know but which can be independently determined by maximizing the entropy with respect to $N$. When both $N$ and $M / M_{p}$ are large, one readily finds, to leading order [7],

$$
S \approx 0.962 \times \frac{\mathcal{A}}{4}
$$

in units of Planck area, which agrees well with the Bekenstein-Hawking value.

In order to describe Hawking radiation [8], we assume the mass function to be a monotonically increasing function of $x=r / l_{p}$. This then encodes information about the black hole as well as the emitted radiation, and Eqn. (4) is to be solved for a given shell, with some assumed mass function. One is interested in the asymptotic solution as $z \rightarrow \infty$, and in the near horizon, $z=1$, solution to this equation. The asymptotic solution which describes outgoing waves on $\mathcal{I}^{+}$is found to be

$$
\Psi^{\infty}=\prod_{j} e^{-\frac{i \epsilon_{j}}{\hbar}\left(\tau_{j}-\frac{2 F_{j}}{c} \sqrt{z_{j}}+\frac{i \hbar}{4 F_{j}} \ln z_{j}\right)}
$$

$\left(\epsilon_{j}=\hbar \omega_{j}\right)$, and the near horizon solution which describes outgoing shells scattered near their horizons is

$$
\Psi^{h o r}=\prod_{j} e^{-\frac{i \epsilon_{j}}{\hbar}\left(\tau_{j}+\frac{F_{j}}{c} \ln |z-1|+\frac{i \hbar}{4 \epsilon_{j}} \ln \left|z_{j}-1\right|\right)} .
$$

If in these two wave functionals the last, $\hbar-$ dependent, term is dropped from the exponent, they coincide exactly with the WKB approximation. Hawking radiation is inferred by calculating the shell by shell Bogoliubov coefficient

$$
\beta\left(\omega, \omega^{\prime}\right)=<\Psi_{\omega^{\prime}}^{\infty \dagger} \mid \Psi_{\omega}^{h o r}>
$$

for the WKB wave functionals, giving the expected result

$$
\left|\beta\left(\omega, \omega^{\prime}\right)\right|^{2}=2 \pi^{2} F^{2} \frac{k T_{H}}{\varepsilon} \frac{1}{e^{\frac{\varepsilon}{k T_{H}}}-1}
$$

which is the Hawking spectrum at the Hawking temperature $k T_{H}=\hbar c^{3} / 8 \pi G M$. An exact series solution to (4) gives the same result [9]. The $\hbar$-dependent term in (10) is responsible for modifying the Hawking radiation, and a fresh calculation of the spectrum with this term retained gives the corrected relation

$$
\left|\beta\left(\omega, \omega^{\prime}\right)\right|^{2}=2 \pi^{2} F^{2} \frac{k T_{H}}{\varepsilon} \frac{1}{e^{\frac{\varepsilon}{k T_{H}}}-1} \sqrt{\frac{2 c}{F \omega^{\prime}}}\left[1-\frac{1}{2} \ln \left(\frac{\pi k T_{H}}{\varepsilon}\right)\right] .
$$


This correction cannot be obtained by modifying the Hawking temperature, and renders the radiation non-thermal. Also, its not equivalent to a correction to black hole entropy - corrections to entropy have been computed by various approaches in the past, but they can all be understood as relating to a thermal spectrum, simply modifying the Hawking temperature. The non-thermal correction suggests that unitarity may not break down in quantum gravitational evolution, and information is not lost. Such a conclusion is also suggested by examining the history of a collapsing shell, as described in [10, 11].

The formalism for analysing quantum gravitational collapse presented here will be of significance also for a proper understanding of the naked singularities that arise in spherical classical gravitational collapse. A semiclassical picture for the evaporation of a naked singularity turns out to be inadequate. This is because the outgoing quantum flux as seen by an asymptotic observer diverges on the Cauchy horizon. A closer examination, however, reveals that this semiclassical approximation breaks down very early during the collapse leading to a naked singularity [12] (for astrophysically relevant collapse, this time scale is the collapse time scale - typically of the order of milli-seconds).

This breakdown occurs because the central curvature very quickly approaches Planck scales, beyond which the semiclassical picture is no longer valid. It is also found 12 that the collapsing star emits a negligible amount of energy (of the order of a Planck unit) during the semiclassical phase. Thus essentially the entire star enters its quantum gravitational phase, on a very short time scale, without any significant Hawking emission. This behaviour should be contrasted with that of an evaporating astrophysical black hole, whose semiclassical evaporation phase is much longer than the age of the universe, and the quantum gravity epoch is reached only after the entire star has nearly evaporated. A naked star is thus the first system known to physicists whose complete evolution cannot be understood without quantum gravity, and which reaches its quantum gravitational phase very much within the lifetime of the universe. Whether quantum gravity causes the naked star to explode catastrophically, or whether it settles down into a gentle black hole state, is a question the answer to which is in fact contained in the Wheeler-DeWitt equation (2).

Classical black holes are black, semiclassical black holes are white, but the non-thermal correction suggests that the quantum gravitational black hole is more like grey. (These grey body factors are associated with the horizon, and are completely different from the grey body factors associated with the radiation at infinity; the latter originate in the backscattering against a classical spacetime geometry.) Hawking's pioneering work on black hole evaporation left us with essentially three options, viz., (a) information is indeed lost during 
the process i.e., the evolution is not unitary, or (b) information is not lost, the semiclassical treatment is misleading and the full quantum evolution is in fact unitary, or (c) a remnant that carries with it all the information content of the black hole is left behind. Our result can be seen as making the case for the second and/or third of the above options much stronger. Moreover, our methods can also be used as the starting point for a serious look at quantum gravity as the Cosmic Censor.

\section{References}

[1] Cenalo Vaz, arXiv:gr-qc/0402025, to appear in the Proceedings of QTS3, held the University of Cincinnati, September 10-14, 2003.

[2] M. Bojowald, Gen. Rel. Grav. 35 (2003) 1877.

[3] K. V. Kuchař, Phys. Rev. D50 (1994) 3961.

[4] H.A. Kastrup and T. Thiemann, Nucl. Phys. B425 (1994) 665.

[5] Cenalo Vaz, L. Witten and T. P. Singh, Phys. Rev. D63 (2001) 104020.

[6] J. D. Bekenstein, Lett. Nuovo Cimento 11 (1974) 467.

[7] Cenalo Vaz and Louis Witten, Phys. Rev. D64 (2001) 084005.

[8] S.W. Hawking, Comm. Math. Phys. 43 (1975) 199.

[9] Cenalo Vaz, L. Witten and T. P. Singh, arXiv:gr-qc/0306045, to appear in Phys. Rev. D.

[10] P. Hájíček, C. Kiefer, Nucl.Phys. B603 (2001) 531-554.

[11] P. Hájíček, Nucl. Phys. B603 (2001) 555-577.

[12] T. Harada, H. Iguchi, K. Nakao, T. P. Singh, T. Tanaka and Cenalo Vaz, Phys. Rev. D64 (2001) 041501. 